\title{
CRÓNICA
}

\section{Reunión del Grupo Fomento Social}

(Madrid, 27-29 noviembre 2009)

\section{José J. Romero Rodríguez S.I.'}

Siguiendo la tradición, el encuentro anual del Grupo Fomento Social se celebró, una vez más, en la Casa de Espiritualidad de las Esclavas del Sagrado Corazón, en Madrid (c/ General Martínez Campos), desde el viernes 27 de noviembre por la noche hasta el domingo 29 a mediodía. La asistencia fue similar a la del año anterior: una veintena de personas, en su mayoría jesuitas junto a los ponentes y algunos laicos afines a la espiritualidad ignaciana y/o colaboradores en instituciones de la Compañía de Jesús. También estuvo presente en todas las sesiones el Provincial de la Compañía de Jesús en España (P. Elías Royón) y el P. Fernando Franco, secretario SJ para la justicia social, con sede en la curia jesuita de Roma.

La preparación de la reunión y su desarrollo corrió a cargo del coordinador del Grupo, P. José María Margenat, profesor de Ética y de Pensamiento Social Cristiano de la Facultad de Ciencias Económicas y Empresariales-ETEA de Córdoba y director de la Revista de Fomento Social.

\section{El tema de fondo: laicidad y pluralismo religioso}

"Laicidad y pluralismo religioso, en relación con la misión de la Compañía de Jesús" era el tema que se había seleccionado en el encuentro del año pasado. A

1 Profesor emérito, ETEA. 
la hora de organizar el trabajo sobre el tema, se optó por la siguiente fórmula: una visión más general (ponencia de la mañana) y un punto de debate actual, aunque más reducido (mesa de diálogo de la tarde).

La ponencia de la mañana ("Laicidad en España actual paradigma para el debate cultural y social"), estuvo a cargo de Rafael Díaz-Salazar Martín, profesor de Sociología de la Universidad Complutense ${ }^{2}$. En la misma abordó en primer lugar la realidad de la laicidad en la identidad de España; un análisis histórico permite detectar que existen una serie de problemas irresueltos. Queda pendiente la cuestión de cómo articular un país que es pluralista en muchos conceptos. En este país nuestro tan visceral parece que se consolidan las dos Españas, con unos cuantos "que nos quedamos en tierra de nadie"; de un lado, la España católica, neo-integrista; del otro, una España caracterizada por un laicismo antieclesial y antirreligioso. El ponente aspira a otra salida: una España articulada, más civilizada, capaz de generar una complexión de interculturalidad.

A continuación expuso el idioma y las claves de una cultura de la laicidad. Se trata de una larga lista, en la que destacamos: la defensa de la laicidad del Estado entendida como neutralidad religiosa e ideológica; la crítica de la teocracia y de la hierocracia; la autonomía de la política y la diversificación de las instituciones; el ejercicio de la tolerancia activa y de la libertad de conciencia; la dignidad y legitimidad de la moral autónoma frente a la heterónoma; la autonomía de la vida espiritual; la importancia de la deliberación moral y oposición al relativismo; y, por último, la defensa de la escuela laica.

Siguió una interesante tipología de la correlación de fuerzas sobre la laicidad (especialmente en España) siguiendo dos ejes: el eje de la religión y el de la dominación de clase. En relación al eje de la religión puso de manifiesto que la diversidad de posiciones es mayor de lo que habitualmente se piensa, tanto del lado de los "laicistas" como del de los "católicos". A propósito del eje "de dominación de clase", llamó la atención sobre la escasa relevancia de las posiciones que propugnan un cambio de modelo social en el socialismo español.

Finalmente, el ponente propuso una agenda con numerosos puntos para avanzar hacia un debate cultural y social. Los dos frentes antes mencionados tendrían

${ }^{2}$ Véanse las obras de su trilogía, imprescindibles sobre estas cuestiones: RAFAEL Díaz-SALAZAR (2001), Nuevo socialismo y cristianos de izquierda, Madrid, Ed. Taurus; Rafael Díaz-Salazar (2007), La España laica, Madrid, Ed. Espasa-Calpe. Rafael Díaz-Salazar (2007); Democracia laica y religión pública, Madrid, Ed. Taurus. 
que dar pasos significativos en esa línea. Por destacar algunos, nos llamaron la atención: su afirmación de que el laicismo como emancipación tiene que llevar a la democracia económica, cuyo rival no son los obispos ni la iglesia, sino la burguesía liberal; su matizada posición sobre la enseñanza de la religión en la escuela pública; su insistencia sobre la ausencia en España de una información religiosa de calidad ("la peor de Europa"); y, por abreviar, su llamada a un "Concilio Vaticano II" ... de la laicidad.

El debate de la mañana, muy participativo (contamos hasta 16 intervenciones de los asistentes, seguidas de comentarios del ponente), abordó un amplio abanico de temas. Destacaríamos lo relativo al modelo de presencia social y política de la Iglesia en España, con interesantes alusiones a otros modelos como el norteamericano.

\section{La mesa de diálogo}

La mesa de diálogo de la tarde tomó como punto de partida otra ponencia sobre el tema "La laicidad en la izquierda española", a cargo de ANTONIO GARCíA SANTESMASES, profesor de Filosofía Política de la UNED, que fue diputado del PSOE y portavoz de Izquierda Socialista, y participante habitual del Foro del Hecho Religioso ${ }^{3}$.

Partió de la afirmación de que nos encontramos en la fase final del proceso de la transición y que se está produciendo de hecho una reconstrucción histórica de los supuestos de la transición empezando por el propio pacto constitucional y siguiendo por los Acuerdos con la Santa Sede.

Hizo un recorrido pormenorizado sobre el desarrollo de los principales acontecimientos que han marcado el tema de la laicidad a lo largo del periodo democrático, antes y a partir del gobierno de Rodríguez Zapatero. Concluyó con una interesante doble agenda:

- Cuestiones en las que es muy difícil ponerse de acuerdo (temas relativos a derechos civiles: aborto, formas de familia, etc.; tema de la escuela y de la enseñanza confesional de la religión).

\footnotetext{
${ }^{3}$ Buena parte de las ideas expuestas pueden encontrarse en su obra: ANTONIO GARCíA SANTESMASES (2007), Laicismo, agnosticismo y fundamentalismo. Madrid, Ed. Biblioteca Nueva, 273 pp.
} 
- Por el contrario, hay algunas cuestiones en las que sería posible el acuerdo y mediante el debate y el diálogo, evitando la polarización: la cuestión de la nación y el Estado; la reconstrucción también de la historia; en política internacional avanzar hacia una verdadera alianza de civilizaciones, erradicando elementos perniciosos de la religión; más aún sería posible alcanzar un consenso acerca del modelo social europeo.

La intervención fue seguida de un coloquio, menos extenso que el de la mañana, pero igualmente intenso e interesante.

\section{Propuesta e informes sobre diversas actividades}

El resto de la tarde del sábado fue completado con varias propuestas y algunas informaciones, entre ellas acerca de una posible mesa de opinión social de los jesuitas españoles: presentada por LIORENCC PUIG y José Ma MARGENAT, la mesa es un subproducto del encuentro de Los Negrales a propósito de la intervención de F. Franco sobre los retos del sector social: cómo coordinar de alguna manera la reflexión social y la incidencia; que el trabajo social, además de acción directa, tenga reflexión detrás, aprovechando los recursos de la Compañía. Algo así como una "Mesa de coordinación de investigación social e incidencia o mesa de generación de opinión". El objetivo principal sería coordinar la información, que los centros sociales proporcionen información sobre lo que les preocupa, que se dé una conexión de los centros sociales entre sí y con otros lugares de reflexión (centros fe-cultura, fe-justicia, revistas, etc.), y que se promueva la difusión y la incidencia. Los tres subsectores (migraciones, jóvenes en riesgo, cooperación, "otros") estarían asimismo implicados.

Pep MÀrIA aporta información sobre el Congreso de Eurojess (grupo de jesuitas europeos que trabajan en ciencias sociales), que tuvo lugar en el Colegio Mayor Loyola de Granada de 26 al 29 de agosto de 2009, con unos 60 participantes. El tema del encuentro, que calificó de excelente tanto por el contenido como por las visitas culturales a Granada y Córdoba, fue "Poblaciones musulmanas en sociedades europeas".

ESTEBAN VELÁZQUEZ informó sobre el proceso del importante congreso del Foro Social Temático Español, organizado en el contexto de la carta de 14 principios del Foro Social Mundial, sobre el tema "Espiritualidades y éticas para otro mundo mejor posible", que tuvo lugar en Sevilla, Palacio de Congresos, 10-12 octubre 2009. Es el primer foro social temático sobre el tema a nivel mundial. 
La mañana del domingo comenzó con la información sobre dos iniciativas en el sector social que están en marcha en estos momentos. FerNANDO FRANCO, director Secretario de Justicia y Ecología de la Compañía, con sede en Roma, informó sobre el trabajo en "advocacy" y redes del sector social de la Compañía de Jesús en el mundo, concretado en la Red de Incidencia Ignaciana ("Ignatian advocacy Network"-IAN) 4 . Comenzó explicando las razones por las que se estaba trabajando en la constitución de estas redes. Enumeró las redes temáticas en marcha, aunque con grado dispar de avance: migraciones; educación; ecología; gobernanza de recursos naturales; fundamentalismo religioso; paz y derechos humanos; ayuda al desarrollo. Cada una de ellas está liderada por alguna institución importante en el área. Por último, detalló los modelos de trabajo en red que se están aplicando según los casos

HIGINIO PI hizo un breve relato de las III Jornadas sociales de la Compañía de Jesús en España, celebradas en Los Negrales en mayo de 2009. Participaron unas 100 personas, la mitad de ellas laicos/as, en un ambiente muy gratificante. Los temas objeto de ponencia fueron tres, aparte del discurso inicial de EĹAs RoYón, provincial de España: Gabriel Pérez AlcalÁ, sobre "Situación económica actual"; Álvaro Alemany y PatXI Álvarez sobre "Nuestra historia de 10 años" (refiriéndose a la historia del sector social de la Compañía en España); y Fernando Franco sobre "Los retos de la Congregación General 35 de la Compañía". La Comisión Interprovincial de Apostolado Social presentó el "Documento de identidad y misión" para el sector, centrado, sobre todo, las obras sociales de la Compañía en España. Cada presentación fue seguida de trabajo en grupos. Un grupo relator recogía y resumía los productos de los trabajos grupales. Se prepara un libro de unas 100 páginas con todo el material de las Jornadas.

\section{Asamblea estatutaria del Grupo}

Finalmente tuvo lugar la Asamblea estatutaria del Grupo. Se procedió, en primer lugar, a un intercambio de pareceres y propuestas sobre el propio grupo Fomento Social. Se confirma la orientación del grupo, como un espacio de encuentro de jesuitas y laicos que trabajan en temas sociales y que quieren reflexionar sobre lo que la identidad de la Compañía significa para este trabajo. Se pidió más presencia de laicos y de jóvenes.

\footnotetext{
${ }^{4}$ Cfr. Fernando Franco (2009), "Redes de Advocacy Ignaciana (IAN) El Modelo Emergente": Promotio
} lustitiae 102 2009/2, pp. 32-36.

Revista de Fomento Social 65 (2010) 
El Encuentro terminó con la elección de tema para la XXIII reunión (noviembre de 2010, 26-28). Previo debate se decidió tratar el tema: "Europa: proyecto, incertidumbre, ampliaciones". Se hicieron también algunas primeras propuestas de posibles ponentes. 